\title{
Determinantes sociais como caminho para promover saúde no pré-natal: percepção dos profissionais da atenção primária*
}

Social determinants as a way to promote health in prenatal care: perception of primary care professionals Determinantes sociales como vías para promover la salud durante el prenatal: percepción de los profesionales de la atención primaria

\section{Carolina Gabriele Gomes da Rocha ${ }^{\mathrm{I}}$, Ivonete Teresinha Schülter Buss Heidemann ${ }^{\mathrm{II}}$, Jeane Barros de Souza $^{\text {III }}$, Michelle Kuntz Durand ${ }^{\mathrm{IV}}$, Kamila Soares Maciel ${ }^{\mathrm{V}}$, Marli Terezinha Stein Backes ${ }^{\mathrm{VI}}$}

\begin{abstract}
Resumo: Objetivo: compreender a percepção dos profissionais da atenção primária acerca da influência dos determinantes sociais na promoção da saúde das gestantes no pré-natal. Método: estudo qualitativo, tipo ação participante, fundamentado no Itinerário de Pesquisa de Paulo Freire, desenvolvido por meio da Investigação Temática, Codificação e Descodificação e o Desvelamento Crítico, que consiste na análise dos dados. Realizaram-se dois Círculos de Cultura em uma Unidade de Saúde, entre julho e outubro de 2019, com 18 profissionais da saúde. Resultados: investigaram-se dois temas: um reflete os determinantes envolvidos no pré-natal; e o outro, discute o cuidado no prénatal e os determinantes como caminho para promover saúde. Desvelaram a relevância de conhecer os determinantes envolvidos no cotidiano das gestantes e que devem ser considerados integralmente. Conclusão: são múltiplas as barreiras enfrentadas pelas gestantes no pré-natal, sendo necessário o seu fortalecimento com cuidados promotores de saúde e articulado aos determinantes sociais.
\end{abstract}

Descritores: Promoção da Saúde; Determinantes Sociais da Saúde; Atenção Primária à Saúde; Cuidado Pré-Natal; Equipe de Assistência ao Paciente

Abstract: Objective: to understand the perception of primary care professionals about the influence of social

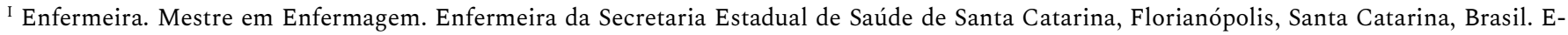
mail: carolinagabriele.r@gmail.com, Orcid: https://orcid.org/0000-0003-4150-9332

II Enfermeira. Pós Doutora em Enfermagem de Saúde Pública. Docente da Universidade Federal de Santa Catarina, Florianópolis, Santa Catarina, Brasil. E-mail: ivonete.heidemann@ufsc.br, Orcid: https://orcid.org/0000-0001-6216-1633

III Enfermeira. Pós Doutora em Enfermagem. Docente da Universidade Federal da Fronteira Sul. Florianópolis, Santa Catarina, Brasil. E-mail: jeane.souza@uff.edu.br, Orcid: https://orcid.org/0000-0002-0512-9765

IV Enfermeira. Pós Doutora em Enfermagem. Docente da Universidade Federal de Santa Catarina, Florianópolis, Santa Catarina, Brasil. E-mail: michakd@hotmail.com, Orcid: https://orcid.org/ 0000-0003-3660-6859

VGraduanda em Enfermagem da Universidade Federal de Santa Catarina. Florianópolis, Santa Catarina, Brasil. E-mail: kmaciel153@gmail.com, Orcid: https://orcid.org/0000-0002-0793-1508

VIEnfermeira. Doutora em Enfermagem. Docente da Universidade Federal de Santa Catarina, Florianópolis, Santa Catarina, Brasil. E-mail: marli.backes@ufsc.br, Orcid: https://orcid.org/0000-0003-3258-359X

*Manuscrito extraído de dissertação "Determinantes Sociais da Saúde no cuidado pré-natal: um olhar multiprofissional na atuação da Atenção Primária”, Programa de Pós-Graduação em Enfermagem, Universidade Federal de Santa Catarina, 2020.
} 
determinants in promoting the health of pregnant women in prenatal care. Method: qualitative study, participant action type, based on Paulo Freire's Research Itinerary, developed through Thematic Investigation, Codification and Decoding and Critical Unveiling, which consists of data analysis. Two Culture Circles were held in a Health Unit, between July and October 2019, with 18 health professionals. Results: two themes were investigated: one reflects the determinants involved in prenatal care, and the other discusses prenatal care and determinants as a way to promote health. They revealed the relevance of knowing the determinants involved in the daily lives of pregnant women and which should be considered in full. Conclusion: there are multiple barriers faced by pregnant women in prenatal care, and it is necessary to strengthen these women with health-promoting care and linked to social determinants.

Descriptors: Health Promotion; Social Determinants of Health; Primary Health Care; Prenatal care; Patient Care Team

Resumen: Objetivo: comprender la percepción de los profesionales de la atención primaria sobre la influencia de los determinantes sociales en la promoción de la salud de mujeres embarazadas durante el prenatal. Método: estudio cualitativo, tipo acción participante, basado en el Itinerario de Investigación de Paulo Freire, desarrollado mediante Investigación Temática, Codificación y Decodificación, y el Desvelamiento Crítico, que consiste en el análisis de los datos. Se realizaron dos Círculos de Cultura en una Unidad de Salud, entre julio y octubre de 2019, con 18 profesionales de la salud. Resultados: se investigaron dos temas: uno refleja los determinantes involucrados en el prenatal; y el otro discute la atención prenatal y los determinantes como vías para promover la salud. Desvelaron la relevancia de conocer los determinantes involucrados en el cotidiano de las embarazadas y que deben ser considerados íntegramente. Conclusión: las mujeres embarazadas se enfrentan a múltiples barreras durante el prenatal, haciéndose necesario su fortalecimiento con cuidados promotores de la salud y articulados a los determinantes sociales.

Descriptores: Promoción de la Salud; Determinantes Sociales de la Salud; Atención Primaria de Salud; Atención Prenatal; Grupo de Atención al Paciente

\section{Introdução}

A promoção da saúde é uma concepção que provoca ambiguidades e contradições. Prevalecem dois discursos distintos: um, com ênfase na modificação dos comportamentos individuais; e outro, que busca avançar para uma perspectiva libertadora, pelo fortalecimento do conhecimento de diferentes significados e conceitos, bem como múltiplas ações e perspectivas. ${ }^{1}$

Em 1986 foi realizada I Conferência Internacional sobre Promoção da Saúde, quando foi divulgada a Carta de Ottawa que conceitua promoção da saúde como o processo que busca possibilitar que as pessoas e a comunidade aumentem o controle e a melhoria da saúde. Nesta carta foram estabelecidas cinco estratégias prioritárias para atuação, as quais são: implementação de políticas saudáveis; criação de ambientes favoráveis à saúde; reorientação dos serviços de saúde; reforço da ação comunitária; e, desenvolvimento de habilidades pessoais. ${ }^{2-3}$

No Brasil, após 30 anos da criação do Sistema Único de Saúde (SUS), pode-se afirmar que 
ainda há um longo caminho para superar o modelo centrado na doença. As ações promotoras de saúde ainda não foram capazes de solidificar o modo de produzir e enfrentar os Determinantes Sociais da Saúde (DSS) que envolvem o processo saúde-doença. ${ }^{4-5}$

Nessa perspectiva, a promoção da saúde precisa ter como foco das ações sanitárias os DSS, que incluem o indivíduo e seu estilo de vida, as redes sociais e comunitárias, a oferta de serviços de saúde, educação, habitação e saneamento, além das condições socioeconômicas, culturais e ambientais. Compreendem-se os DSS como os fatores que influenciam as condições de saúde da população e estão dispostos aos níveis individual, social, econômico, cultural, étnicos/raciais, psicológicos e comportamentais. ${ }^{4}$ Portanto, é relevante a sinergia dos DSS com a promoção da saúde em prol da equidade, para melhor compreensão e endereçamento das iniquidades. ${ }^{4}$

Destaca-se a preocupação quanto ao cuidado pré-natal na Atenção Primária à Saúde (APS), que integra um dos assuntos de maior discussão e investimento público na atualidade. O acolhimento da gestante na APS acontece em espaços individuais e coletivos, como consultas e reuniões de grupo. O fortalecimento do cuidado no pré-natal pelos profissionais da APS pode propiciar intervenções que contribuem para a diminuição de partos prematuros, enfermidades neonatais e a mortalidade infantil. ${ }^{6}$

Assim, entende-se que o atendimento à gestante deva ser multidimensional. Requer ultrapassar os limites dos consultórios e ser realizado não somente pela equipe de Saúde da Família (eSF), mas por toda equipe multiprofissional, incluindo o Núcleo Ampliado de Saúde da Família e Atenção Básica (NASF-AB). ${ }^{7}$ A abordagem multiprofissional no pré-natal favorece a humanização e a integralidade da atenção, que são dispositivos qualificadores do processo de promover a saúde das mulheres durante o período gestacional. Ademais, a integração de conhecimento dos diversos profissionais em uma equipe possibilita intervenções consideradas relevantes na assistência contínua que precede o parto. ${ }^{8}$

O fato é que são múltiplas as barreiras enfrentadas pelas gestantes: desigualdades regionais, sociais e econômicas; dificuldade de acesso aos locais de consultas; incompreensão das 
orientações prescritas; tempo prolongado de espera para consultas; e, a não realização dos exames. ${ }^{9}$ Independente desses fatores determinantes e condicionantes, há uma infraestrutura insatisfatória e número insuficiente de profissionais, que revelam fragilidades para estes enfrentarem os DSS: a desorganização da APS à assistência pré-natal, a falta de consultório específico, além de recursos materiais para consultas e de medicamentos essenciais de uso na gravidez. ${ }^{10}$

Para promover a saúde das gestantes durante o pré-natal, faz-se necessário envolver diferentes atores, de modo a produzir um acompanhamento de excelência, neste período. Para tanto, é essencial conhecer seus perfis, a fim de identificar os DSS que possam interferir no desenvolvimento saudável da gestação, pois essa particularidade dará respaldo à equipe de saúde para desenvolver ações de promoção que proporcionem melhoria da qualidade de vida, ${ }^{8-9}$ o que justifica a relevância desse estudo.

A partir de uma revisão narrativa de literatura, identificaram-se poucos estudos que relacionavam os DSS das gestantes, no contexto do pré-natal na APS. Urgem, assim, esforços dos profissionais de saúde para que seja abordada esta importante temática. Frente ao exposto, o objetivo deste estudo foi compreender a percepção dos profissionais da atenção primária acerca da influência dos determinantes sociais na promoção da saúde das gestantes no pré-natal.

\section{Método}

Trata-se de um estudo com abordagem qualitativa, do tipo ação-participante, ${ }^{11}$ articulado ao Itinerário de Pesquisa de Paulo Freire, que se constitui em três etapas distintas e interligadas entre si, a saber: Investigação Temática; Codificação e Descodificação; e, o Desvelamento Crítico. O Itinerário de Pesquisa de Paulo Freire se desenvolve em formato dinâmico, em espaço denominado de Círculo de Cultura, que é caracterizado por um grupo de indivíduos com algum interesse em comum, que se reúnem para refletir sobre seus problemas, compartilhar vivências, construir uma percepção mais profunda da realidade e estruturar coletivamente ações concretas de intervenção. ${ }^{12-13}$

Desenvolveu-se a pesquisa entre os meses de julho e outubro de 2019. Foram realizados 
5 | Rocha CGG, Heidemann ITSB, Souza JB, Durand MK, Maciel KS, Backes MTS

dois Círculos de Cultura com duração aproximadamente de 90 minutos, cada encontro, os quais ocorreram na sala de reuniões de uma Unidade Básica de Saúde (UBS), de um município do litoral de Santa Catarina, região Sul do Brasil, com o apoio da Secretaria Municipal de Saúde.

Os pesquisadores, com experiência na metodologia e treinamento nesse tipo de abordagem, foram os mediadores neste estudo. Eles já mantinham vínculos com essa UBS - que atende um maior número de gestantes, por terem realizado atividades teóricas e práticas em graduação de enfermagem da Universidade Federal de Santa Catarina (UFSC). Dos profissionais da saúde que trabalhavam nesta UBS, 18 foram os participantes. O coordenador da unidade escolhida foi previamente convidando para a participação dos Círculos de Cultura em dias de reuniões da equipe.

Para critérios de inclusão, elencaram-se: ser profissional vinculado às equipes de atenção primária; e estar presente nos dias de realização dos Círculos de Cultura para participar das discussões e reflexões. Como critérios de exclusão, foram preteridos profissionais afastados por férias ou licença. No primeiro Círculo de Cultura, os pesquisadores compartilharam acerca dos objetivos do estudo e os participantes assinaram o Termo de Consentimento Livre e Esclarecido.

Para a Investigação Temática, primeira etapa do Itinerário de Pesquisa de Freire, a mediadora partiu da percepção dos profissionais sobre promoção da saúde e a relação com os determinantes sociais no pré-natal. Estimulou-se o debate por meio de uma questão disparadora: qual a influência dos DSS na promoção da saúde para você? Foram disponibilizadas tarjetas para cada participante descrever o significado da temática proposta. Todos puderam compartilhar a sua reflexão sobre promoção da saúde e levantaram várias questões, organizando dois temas geradores para discussão no próximo Círculo de Cultura: 1) DSS envolvidos no pré-natal; 2) Cuidado no prénatal e os DSS como caminho para promover saúde.

No segundo Círculo de Cultura, para instigar o diálogo e torná-lo mais dinâmico, interativo e concreto, optou-se por percorrer as etapas do Itinerário de Pesquisa de Paulo Freire a partir de uma analogia com as partes de um peixe, representando o contexto de atuação dos profissionais, ou seja, o contexto litorâneo, conforme a Figura 1. Nessa analogia, refletiu-se acerca das partes de um 
peixe, que estão interligadas entre si, tal como o Itinerário de Pesquisa de Paulo Freire.

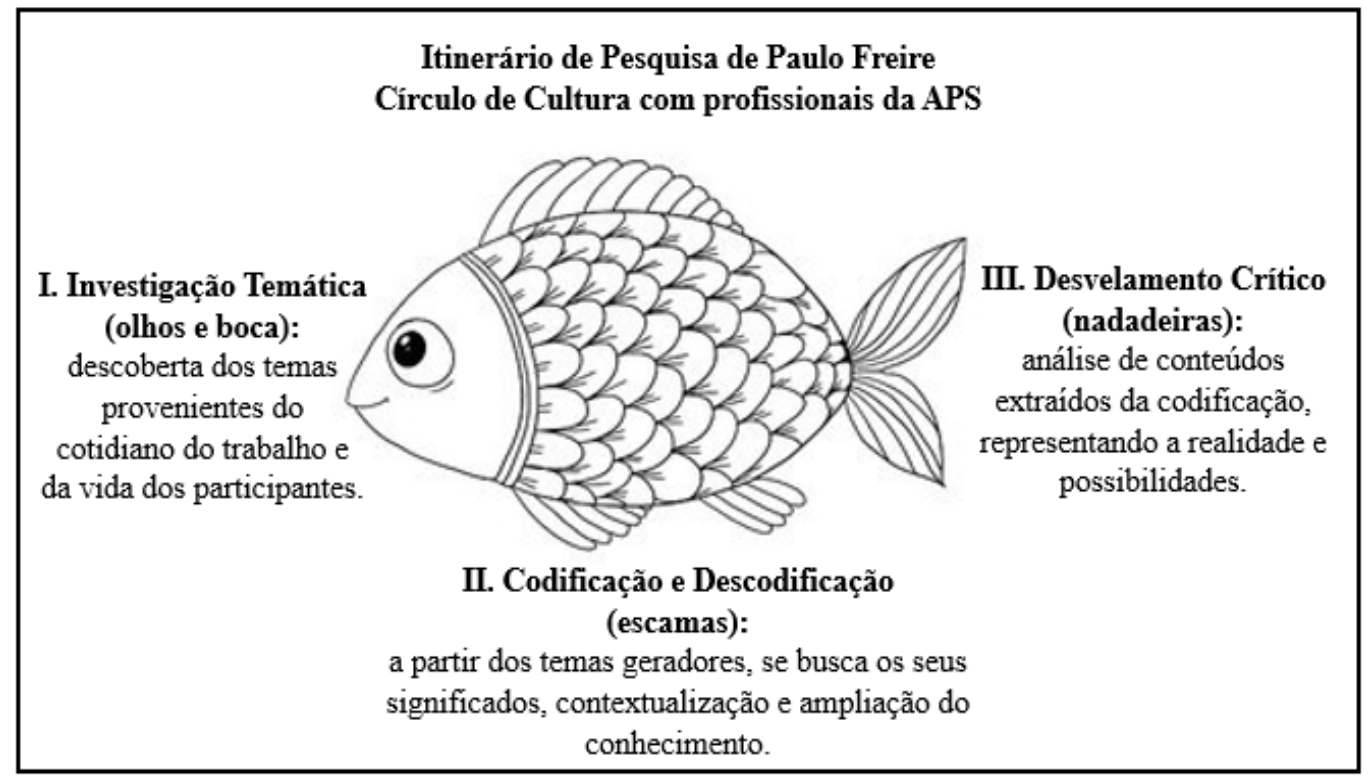

Figura 1: Itinerário de Pesquisa: analogia com o peixe

Fonte: Imagem adaptada pelas autoras (https://pt.clipart.me/istock/coloring-book-with-fish-674455)

Para resgatar e validar os dois temas geradores levantados no primeiro Círculo de Cultura, expôs-se no chão um tecido azul, simbolizando o mar da vida. No centro do tecido foi colocado um barco, com a imagem de uma gestante. As duas proposições foram simbolizadas, cada uma, por um grande peixe desenhado em uma cartolina, acompanhado de peixes menores contendo as mesmas cores, com a descrição de um termo representativo, discutido no primeiro encontro.

A mediadora releu os dois temas e suas representações e convidou os participantes a voltarem os olhos para a gestante no mar da vida, a fim de refletir sobre os fatores que influenciam o bem-estar e a promoção da saúde durante o pré-natal, instigando-os à discussão sobre os DSS. Para a Codificação e Descodificação dos dois temas geradores, os participantes foram divididos em dois grupos (Lilás e Verde). Cada grupo recebeu um nome e um cartaz com a figura de um peixe, contendo uma questão para reflexão. O Grupo Lilás refletiu sobre: como os DSS influenciam na qualidade de vida durante o pré-natal? O grupo Verde analisou acerca de: como os DSS influenciam no cuidado durante o pré-natal?

Para problematizar as questões levantadas, a mediadora compartilhou o modelo conceitual 
7 | Rocha CGG, Heidemann ITSB, Souza JB, Durand MK, Maciel KS, Backes MTS

de Dahlgren e Whitehead ${ }^{4}$ em uma cartolina, com vistas a representar de maneira clara os DSS. Os grupos debateram intensamente e, em seguida, apresentaram suas percepções para o grande grupo, codificando e descodificando os dois temas geradores.

O Desvelamento Crítico ocorreu simultaneamente, conforme prevê o processo analítico de Paulo Freire, fundamentado no referencial dos DSS. Etapa tradicionalmente chamada de análise dos dados, é um processo contínuo com o envolvimento dos participantes que compõem os Círculos de Cultura. É também denominado de fase de problematização, isto é, momento da construção do conhecimento por meio da revelação de conceitos emergentes. Representa a tomada de consciência em que a situação vivenciada e compartilhada na fase da codificação é problematizada e descodificada, desvelando um novo olhar e gerando o processo de ação-reflexão-ação. ${ }^{12-13}$

Para o registro dos temas foi realizada gravação de áudio, previamente autorizada pelos participantes, com o objetivo de registrar os encontros na íntegra. A pesquisa foi aprovada pelo Comitê de Ética em Pesquisa da UFSC, com parecer número 3.253 .685 e CAAE 10432719.8.0000.0121 na data de 09 de abril de 2019, sendo cumpridas as determinações das Resoluções 466/12 e 510/2016 do Conselho Nacional de Saúde. Para garantir o anonimato, os participantes foram identificados com nomes de praias do litoral catarinense.

\section{Resultados}

Os 18 profissionais da saúde que integraram o estudo eram: um médico, três enfermeiras, um cirurgião-dentista, uma auxiliar de saúde bucal, quatro agentes comunitários de saúde, uma profissional de educação física, um fisioterapeuta, três técnicas de enfermagem e quatro nutricionistas. A faixa etária dos participantes variou de 23 a 51 anos e o tempo de atuação na APS era de um mês a 22 anos.

Os dois temas geradores que emergiram para discussão nos Círculos de Cultura foram: 1) Condições de vida das gestantes como Determinantes Sociais da Saúde; 2) Atenção pré-natal como Determinante Social da Saúde. 
Determinantes sociais como caminho para promover saúde no pré-natal ... $\mid 8$

\section{Condições de vida das gestantes como Determinantes sociais da saúde}

Os participantes apontaram que os DSS podem influenciar negativa ou positivamente na adesão e no desenvolvimento do pré-natal e desvelaram o gênero como aspecto balizador. Refletiram sobre a realidade enfrentada pelas mulheres do mundo atual e como isso interfere na qualidade de vida das gestantes. Além do gênero, a idade foi destacada pelos profissionais, que despertou diálogos sobre as gestantes adolescentes, sendo um fator preocupante e desafiador, pois o estilo de vida destas pode influenciar negativamente a qualidade de vida durante o pré-natal:

Em ser mulher já um influenciador, muitas vezes, pela dupla jornada de trabalho: trabalha em casa, trabalha fora, tem o cuidado com a família. (Campeche)

Nós refletimos sobre vários fatores determinantes. Por exemplo, se é uma gestante adolescente, como ela irá lidar com essa situação na gestação, com o estilo de vida que ela tem. (Ingleses)

A situação socioeconômica, como trabalho e renda, foi apontada como influenciadora na qualidade do pré-natal, pois afeta em outros fatores, como a alimentação e o lazer, que por sua vez repercutem na qualidade de vida das gestantes:

A situação econômica da gestante, uma situação de desemprego, alguma dificuldade financeira, isso também influencia em vários aspectos, na alimentação, lazer, cultura. Então são vários aspectos que podem influenciar na qualidade de vida dela. (Do Rosa)

Os participantes destacaram o tempo como algo que influencia constantemente no caminho para promover a saúde. Refletiram e perceberam que o tempo é socialmente determinado, sendo permeado por questões sociais, econômicas e de gênero:

O tempo disponível é socialmente determinado. O tempo disponível para si mesmo, para se cuidar, depende de fatores superindividuais, como raça, cor e classe social. No Brasil ser mulher negra e pobre significa ter menos tempo para si, significa morar mais longe do trabalho, receber menos, ter ocupações menos salubres. Ter outros filhos também vai limitar o que é possível para si mesmo, condição de trabalho e situações mais dramáticas, como desemprego. (Ipanema)

O suporte da família e do companheiro durante a gestação também foram levantados pelos participantes, que destacaram que as gestantes que possuem essa rede de apoio fortalecida demonstram um diferencial em relação àquelas que não a têm, interferindo diretamente na qualidade de vida durante o pré-natal:

Se a rede de apoio está fragilizada, como será o cuidado dela? Ter alguém para conversar, 
9 | Rocha CGG, Heidemann ITSB, Souza JB, Durand MK, Maciel KS, Backes MTS

alguém para acompanhar as consultas, acreditamos ser um diferencial. (Lagoinha do Leste)

Desvelou-se que, são inúmeros os fatores que interferem na qualidade de vida das mulheres durante a gestação, os quais perpassam a jornada de trabalho, renda, idade e rede de apoio e precisam ser conhecidos e compreendidos pelos profissionais de saúde, de modo a promover a saúde com base no referencial dos DSS.

\title{
Atenção pré-natal como Determinante Social da Saúde
}

Os profissionais refletiram acerca da importância de não culpabilizar as gestantes, considerando sempre os fatores que condicionam o seu bem-estar:

\begin{abstract}
A não continuidade do pré-natal por faltas em consultas precisa ser discutida, pois resulta em culpabilização das gestantes pelos profissionais. No momento em que pensamos nos aspectos que limitam as possibilidades de decisão e de opções individuais saímos do discurso de culpabilização das vítimas e, consequentemente, da falácia que a saúde de cada um depende das suas escolhas individuais ou que depende estritamente das suas decisões individuais. (Ponta das Canas)

Tem a ver com a nossa prática no dia a dia. A gente conversou sobre algumas questões aqui, por exemplo, faltou uma consulta de pré-natal na chuva, no sistema aparece assim: "Você vai querer atender essa pessoa, mesmo ela faltando?” Então a gente culpabiliza se a gente não discute qual o nosso papel aqui na UBS, que é atender o outro. E como que a gente olha esse ser humano. (Rififi)
\end{abstract}

Para os participantes do estudo, ter empatia com a gestante e entender os reais motivos que as levam a tomar decisões durante o pré-natal são atos imprescindíveis para qualificar o atendimento prestado e a promoção à saúde da mulher e do bebê:

Como eu gostaria de ser atendida se eu estivesse grávida e fosse a UBS? Como que eu gostaria de ser atendida se eu chegasse mal com uma série de problemas sociais, problemas emocionais? Será que eu gostaria que alguém me olhasse feio ou eu gostaria que alguém falasse "Bom dia! Em que eu posso te ajudar". (Açores)

Os participantes refletiram sobre o cuidado prestado durante o pré-natal e destacaram a relevância de ser centrado na gestante e na sua família, bem como nas demandas que essa mulher apresenta, evitando o cuidado excessivo, que pode acarretar em conflitos durante esse período:

Os determinantes sociais são fundamentais para elaboração de um plano de cuidado e assistência pré-natal, desde que sejam centrados na pessoa. Quando a gente vai atender uma mulher ou uma familia, qual a situação econômica, social e ambiental da construção dessa maternidade? Vamos ter que levar em conta aquilo que aquela mulher nos traz e isso reflete muito em quanto de cuidado que essa mulher pode suportar, o quanto que ela veio buscar, para que eu não seja excessiva em coisas que talvez eu não atinja realmente ela. Então, a 
Determinantes sociais como caminho para promover saúde no pré-natal ... | 10

construção em cima da bagagem cultural que essa mulher traz, das condições de vida e de trabalho, das redes sociais que ela ocupa, de estilo de vida que ela leva, as questões alimentares, onde ela vive, como ela vive, a rede de apoio social que ela tem, habitação. Enfim, todos os determinantes sociais vão fazer com que eu procure dentro da assistência focar naquilo que é necessário e interessante. (Pântano do Sul)

Além disso, os profissionais dialogaram sobre a necessidade de conhecer a realidade da gestante, desde o início do pré-natal, buscando conhecer o significado desta gestação e da maternidade para ela. Outro aspecto de discussão foi a construção em conjunto (profissionalgestante-família) de um plano de cuidado, que leve em consideração os DSS como uma possibilidade para promover a saúde e o empoderamento feminino, durante o processo de gestar:

Então, por exemplo, uma mulher que chega a iniciar um pré-natal no consultório, acho que a primeira abordagem é a gente tentar entender quem é essa mulher, como que ela nasceu, como que ela construiu a vida dela, como que é essa maternidade, o que essa maternidade significa para ela e a partir disso, tornar possível dentro dessa história, montar uma assistência, não no que eu acho que é certo, mas no que faz sentido para ela. (Galheta)

Esses dias dei um exemplo bem claro sobre DSS. Eu atendi uma paciente que tem um modo de vida mais alternativo e outra com uma crença diferente, por exemplo, uma evangélica e uma budista. Eu não sou nem budista, nem evangélica, mas como que eu posso fazer que isso faça sentido para ela, para que ela possa sair dali depois de nove meses com esse bebê no colo, e com a sensação de que ela está constituindo a maternidade de uma forma que ela seja empoderada para isso, que ela tenha o poder, para entender que o processo é dela e não meu. A construção é nossa!'(Açores)

O diálogo nos Círculos de Cultura estimulou a reflexão dos DSS no contexto da gestação e interface familiar, social e cultural. Esses fatores apareceram como situações que influenciam a gestação e que devem ser considerados quando o profissional realiza o acompanhamento pré-natal, como um caminho para promover a saúde da mulher no seu processo de gestar.

\section{Discussão}

A promoção da saúde consiste em um conjunto de estratégias, no âmbito individual e coletivo, que visa atender às necessidades sociais de saúde e à melhoria da qualidade de vida. ${ }^{5}$ No entanto, a efetividade desses princípios depende, em nível macro, da atuação sobre os DSS e, em nível micro, da atuação sobre a pessoa, família e comunidade. ${ }^{4}$

Cabe salientar que o modelo Dahlgren e Whitehead ${ }^{4}$ descreve os determinantes sociais que 
11 | Rocha CGG, Heidemann ITSB, Souza JB, Durand MK, Maciel KS, Backes MTS

influenciam a saúde. No centro desse modelo se encontram os indivíduos com as suas características de idade, gênero e fatores hereditários. Ao observar os depoimentos dos profissionais da APS, eles apontaram as idades das gestantes, citando sobre a gravidez na adolescência, bem como abordaram a fragilidade do sexo feminino, e da vulnerabilidade econômica das mesmas, principalmente a situação da mulher de cor negra.

No primeiro nível do modelo de Dahlgren e Whitehead ${ }^{4}$ estão os fatores relacionados com os estilos de vida. Os participantes desse estudo evidenciaram essa situação, destacando a falta de tempo no viver da mulher gestante, que interfere nas suas escolhas, conforme o seu comportamento e maneira de viver. No segundo nível se encontram as redes de apoio sociais e comunitárias, sendo que os profissionais da saúde abordaram sobre a relevância do amparo dos familiares e companheiro da mulher gestante no caminho da promoção da saúde no pré-natal.

O terceiro nível ilustra os macrodeterminantes, relacionados com os fatores econômicos, ambientais e culturais da sociedade em geral. Nesse aspecto, os participantes do estudo evidenciaram as condições econômicas, em que destacaram o desemprego, educação e pobreza da mulher, que podem interferir nas suas condições de saúde. Também lembraram de lazer, moradia e alimentação, mas nada citaram acerca da situação de água, esgoto e produção agrícola dos alimentos, que é mencionado no modelo de Dahlgren e Whitehead. ${ }^{4}$

As condições sociais possuem uma significativa influência sobre hábitos e comportamentos do indivíduo. Somado a isso, influenciam no conhecimento, na percepção e na capacidade de autogerenciamento da condição de saúde. ${ }^{14}$ A partir de um amplo olhar, pode-se perceber que o reconhecimento das desigualdades socioeconômicas e a identificação da dimensão destas, são imprescindíveis para a promoção de políticas públicas que possam suprimir estas diferenças, ${ }^{15}$ principalmente no que tange às populações mais vulneráveis.

A mulher, ao gestar, pode-se tornar vulnerável diante dos aspectos sociais e demográficos que a envolvem, que influenciam nas condições de saúde. A gestação consiste em um processo fisiológico natural e compreende uma sequência de alterações físicas, psicológicas e sociais 
Determinantes sociais como caminho para promover saúde no pré-natal ... | 12 específicas, que demandam adaptações não somente no corpo, mas especialmente, na vida feminina. ${ }^{15}$ Durante este processo de intensas mudanças, espera-se que a gestação seja uma experiência positiva. Para que isso aconteça, a relação profissional/usuária, estabelecida durante o pré-natal, precisa ser baseada no diálogo, a fim de que as informações e as orientações sobre os cuidados em saúde possam ser vistas como um diferencial que contribua para o alcance da qualidade da assistência. ${ }^{16}$

No entanto, muitos são os desafios na APS, para o alcance do pré-natal qualificado, pois se entende a maternidade como uma construção social. A proteção da gestante ainda se encontra ameaçada, marcada por um histórico de negligências nos âmbitos sanitário, assistencial e político. As gestantes que buscam o cuidado pré-natal na APS podem-se deparar(-se) com dificuldades relacionadas à disponibilidade, aos custos diretos e indiretos da assistência e à relação que se estabelece com os serviços. Neste sentido, entender que as peculiaridades envolvidas no acesso ao cuidado pré-natal colaboram para a reorganização dos serviços de saúde. ${ }^{17}$

Ressalta-se que as oportunidades sociais não são igualmente oferecidas, pois a mulher possui longa jornada de trabalho devido às inúmeras ocupações das quais se apropria, o que pode gerar estresse e sobrecarga física, somado às desigualdades de gênero para conciliar as tarefas. ${ }^{18}$ As jovens e menos favorecidas, que se encontram desprovidas de projetos educacionais e profissionais, sentem o peso das limitações econômicas e a falta de acesso a diversos benefícios sociais, que violam direitos básicos. A gestação na adolescência no Brasil é um dos fatores que as tornam com menores possibilidades de trabalho e de vida digna, sendo necessário desenvolver ações que promovam os seus direitos e condições dignas de vida. Portanto, esse público carece de acompanhamento no pré-natal de maneira acolhedora e humanizada por parte dos enfermeiros e demais profissionais da APS, com vistas a enfrentar as vulnerabilidades que as cercam. ${ }^{19}$

Fatores como nível de escolaridade e percepção da necessidade de tratamento influenciam na obtenção de conhecimentos e de cuidados em saúde. Indivíduos com maiores privações sociais possuem crenças sobre sua saúde e menor percepção da manutenção de cuidados essenciais. Nesse 
13 | Rocha CGG, Heidemann ITSB, Souza JB, Durand MK, Maciel KS, Backes MTS

contexto, a saúde é um conceito que expressa crenças sociais e populacionais, que por sua vez, são influenciadas por valores culturais e demográficos, que decorrem de sua relação com o território e suas características. ${ }^{10-15}$

Os profissionais da APS se corresponsabilizam pela saúde dos indivíduos do território abrangido pela equipe, apropriando-se das demandas e do contexto de saúde, social, histórico, cultural e ambiental para o planejamento de ações. Por meio dessa atuação, os laços se tornam estreitos entre profissionais e usuárias, o que favorece a constituição de vínculos e atuação de forma ampla, considerando os fatores determinantes e condicionantes, o que colabora para o desenvolvimento do cuidado pré-natal. A APS deve fortalecer seu papel como coordenadora da Rede de Atenção à Saúde e do cuidado, com vistas a possibilitar o acesso e a continuidade do acompanhamento pré-natal, buscando, principalmente, mediar o empoderamento das gestantes, para que sejam protagonistas de sua própria história. ${ }^{20-21}$ Cabe enfatizar que, ao buscar valorizar e promover a saúde das gestantes, os profissionais precisam considerar os DSS, sem culpabilizá-las no processo saúde-doença. ${ }^{22}$

Ademais, torna-se imprescindível que os profissionais estejam sensíveis e atentos para identificar a rede de apoio, atuar em conjunto e alcançar a qualidade durante o pré-natal. A família está diretamente relacionada com os DSS e é percebida como um fator importante neste período, pois constitui um sistema complexo que influencia diretamente sobre todos os membros. É a partir dela que são fornecidos suporte emocional e orientações para construir conceitos, crenças, valores e tradições. As influências geradas pela rede familiar impactam também na forma como os indivíduos se cuidam, como lidam com o processo saúde-doença, o que se estende ao período gestacional, parto e pós-parto. ${ }^{23}$

Desvela-se a importância de os profissionais da saúde construírem um plano de cuidado, em conjunto com as gestantes, considerando as necessidades de saúde e os DSS. Na APS, os profissionais devem buscar conhecer com empatia a história das mulheres, suas interfaces com a gestação, seu contexto de vida e proporcionar momentos de escuta e interação que permitam que 
elas se tornem ativas no cuidado, promovendo o empoderamento feminino no processo de gestar.

O cuidado no pré-natal realizado na APS transcende condutas biologicistas no cuidado à saúde, tornando-se fundamental compreender e reconhecer os fatores sociais, econômicos, culturais, étnico-raciais, psicológicos e comportamentais da gestação, para promover a atenção integral. Nesse cenário, a escuta é um excelente recurso para saber quais são as necessidades de cada gestante e, a partir de então, proporcionar-lhe as informações e os cuidados pertinentes. ${ }^{24}$ Assim, torna-se fundamental o desenvolvimento de ações interdisciplinares pelos profissionais que atuam no contexto da atenção primária à saúde. O cuidado no pré-natal requer a realização de atividades que englobem diferentes saberes, que podem ser oriundos dos profissionais da área da saúde como de outros setores da sociedade. ${ }^{25}$

Como limitação do estudo, aponta-se o tempo reduzido para a realização dos Círculos de Cultura, que foram desenvolvidos durante as reuniões da eSF, de modo a otimizar a participação dos profissionais da APS. Destaca-se ainda, o número restrito de estudos relacionados à temática dos DSS no contexto do pré-natal.

\section{Conclusão}

Os participantes debateram as condições de vida das gestantes como DSS, refletindo sobre o atendimento que lhes é prestado na APS. Desvelaram que é fundamental promover o acompanhamento pré-natal a partir de seus modos de ser e viver, de suas famílias e no contexto em que estão inseridas. O conhecimento dos DSS facilita a promoção da saúde da gestante e auxilia na elaboração de um plano de cuidado pré-natal que caminha ao encontro das expectativas e necessidades de cada mulher. A compreensão da influência dos DSS no pré-natal é perceptível entre os profissionais na APS. Por meio deste estudo, os participantes ressaltaram as dificuldades socioeconômicas enfrentadas durante a gestação, a problemática da idade e, ainda, sobre a rede de apoio, as quais repercutem na qualidade de vida e na promoção da saúde dessas mulheres.

A abordagem metodológica escolhida oportunizou momentos de reflexão e de troca de 
15 | Rocha CGG, Heidemann ITSB, Souza JB, Durand MK, Maciel KS, Backes MTS

experiências entre os participantes. O Itinerário de Pesquisa de Paulo Freire propiciou o entendimento das situações sociais e compreensão da realidade, que foram alcançados por meio do diálogo coletivo. Sugere-se o desenvolvimento de outros estudos com metodologias participativas, pois, tornam-se mais amplos, libertadores e com resultados que ultrapassam o âmbito da pesquisa, repercutindo na transformação da realidade. Recomenda-se a realização de novas pesquisas com esta temática, com foco na prática de cuidado e ensino dos profissionais da saúde.

\section{Referências}

1. Heidemann ITSB, Alonso da Costa MFBN, Hermida PMV, Marçal CCB, Antonini FO, Cypriano CC. Health promotion practices in primary care groups. Glob Health Promot. 2019;26(1):25-32. doi: $10.1177 / 1757975918763142$

2. Farias JM, Minghelli LC, Soratto J. Promoção da saúde: discursos e concepções na atenção primária à saúde. Cad Saúde Colet. 2020 set;28(3):381-9. doi: 10.1590/1414-462x202028030351

3. World Health Organization (WHO). Health Promotion [Internet]. Ottawa (CA): WHO; 1986 [cited 2021 Jan 17]. Available from: https://www.who.int/healthpromotion/conferences/previous/ottawa/en/

4. World Health Organization (WHO). Closing the gap in a generation: health equity through action on the social determinants of health [Internet]. Geneva: WHO, Comission on Social Determinants of Health; 2008 [cited 2020 Nov 17]. Available from: https://www.who.int/social_determinants/final_report/csdh_finalreport_2008.pdf

5. Malta DC, Reis AAC, Jaime PC, Morais Neto OL, Silva MMA, Akerman M. Brazil's Unified Health System and the National Health Promotion Policy: prospects, results, progress and challenges in times of crisis. Ciênc Saúde Colet. 2018;23(6):1799-809. doi: 10.1590/1413-81232018236.04782018

6. Schmitt PM, Tomazzetti BM, Hermes L, Hoffmann IC, Braz MM, Martelo NV. A revelação de puérperas na assistência pré-natal em estratégias de saúde da família. Rev Saúde Pesqui. 2018;11(1):129-37. doi: 10.17765/19831870.2018v11n1p129-137

7. Livramento DVP, Backes MTS, Damiani PR, Castillo LDR, Backes DS, Simão AMS. Perceptions of pregnant women about prenatal care in primary health care. Rev Gaúcha Enferm. 2019;40:e20180211. doi: 10.1590/19831447.2019.20180211

8. Santiago CMC, Sousa CNS, Nóbrega LLR, Sales LKO, Morais FRR. Prenatal care and practices developed by the health team: integrative review. Rev Pesq Cuid Fundam. 2017;9(1):279-88. doi: 10.9789/21755361.2017.v9i1.279-288

9. Blakeney EL, Herting JR, Bekemeier B, Zierler BK. Social determinants of health and disparities in prenatal care utilization during the Great Recession period 2005-2010. BMC Pregnancy Childbirth. 2019;19: 390. doi: 10.1186/s12884-019-2486-1 
10. Rocha CRG, Heidemann IHSB, Rumor PCF, Antonini FO, Durand MK, Magagnin AB, et al. Social determinants of health in prenatal nursing consultation. Rev Enferm UFPE On Line. 2019;13:e241571. doi: 10.5205/1981-8963.2019.241571

11. Felcher CDO, Ferreira ALA, Folmer V. Da pesquisa-ação à pesquisa participante: discussões a partir de uma investigação desenvolvida no Facebook [Internet]. 2017 [acesso em 2021 jan 10];12(7):1-18. Disponível em: https://if.ufmt.br/eenci/artigos/Artigo_ID419/v12_n7_a2017.pdf

12. Freire P. Pedagogia do oprimido. 60ª ed. Rio de Janeiro: Paz e Terra; 2016.

13. Heidemann ITSB, Dalmolin IS, Rumor PCF, Cypriano CC, Costa MFBNA, Durand MK. Reflections on Paulo Freire's research itinerary: contributions to health. Texto Contexto Enferm. 2017;26(4):e0680017. doi: 10.1590/0104-07072017000680017

14. Heidemann ITSB, Cypriano CC, Gastaldo D, Jackson S, Rocha CG, Fagundes E. Estudo comparativo de práticas de promoção da saúde na atenção primária em Florianópolis, Santa Catarina, Brasil e Toronto, Ontário, Canadá. Cad Saúde Pública. 2018;34(4):e00214516. doi: 10.1590/0102-311x00214516

15. Barbieri W, Peres SV, Pereira CB, Peres Neto J, Sousa MLR, Cortellazzi KL. Fatores sociodemográficos associados ao grau de conhecimento em saúde bucal de gestantes. Einstein. 2018;16(1):eAO4079. doi: 10.1590/S1679-45082018AO4079

16. Gaíva MAM, Palmeira EWM, Mufato LF. Women's perception of prenatal and delivery care in cases of neonatal death. Esc Anna Nery. 2017;21(4):e20170018. doi: 10.1590/2177-9465-ean-2017-0018

17. Tinoco TF, Acioli S, Mercedes Neto, Silva LA. Mortalidade materna e a prática educativa na atenção primária à saúde. Nursing [Internet]. 2018 [acesso em 2020 nov 18];21(247):2535-41. Disponível em: http://www.revistanursing.com.br/revistas/247/pg89.pdf

18. Costa FA. Mulher, trabalho e família: os impactos do trabalho na subjetividade da mulher e em suas relações familiares. Pretextos [Internet]. 2018 [acesso em 2020 nov 18];3(6):434-52. Disponível em: http://periodicos.pucminas.br/index.php/pretextos/article/view/15986

19. Santos RCAN, Silva RM, Queiroz MVO, Jorge HMF, Brilhante AVM. Realities and perspectives of adolescent mothers in their first pregnancy. Rev Bras Enferm. 2018;71(1):65-72. doi: 10.1590/0034-7167-2016-0444 20. Silva LA, Alves VH, Rodrigues DP, Vieira BDG, Marchiori GRS, Santos MV. The humanization of prenatal care under the pregnant women's perspective. Rev Pesq Cuid Fundam. 2018;10(4):1014-9. doi: 10.9789/21755361.2018.v10i4.1014-1019

21. Souza EVA, Bassler TC, Taveira AG. Health education in the empowerment of the pregnant woman. Rev Enferm UFPE On Line [Internet]. 2019 [cited 2021 Apr 20];13(5):1527-31. Available from: https://periodicos.ufpe.br/revistas/revistaenfermagem/article/view/238437

22. Rowlands G, Shaw A, Jaswal S, Smith S, Harpham T. Health literacy and the social determinants of health: a qualitative model from adult learners. Health Promot Int. 2017;32(1):130-8. doi: 10.1093/heapro/dav093

23. Romagnolo AN, Costa AO, Souza NL, Somera VCO, Benincasa M. A família como fator de risco e de proteção na gestação, parto e pós-parto. Semin Ciênc Soc Hum. 2017;38(2):133-46. doi: 10.5433/16790383.2017v38n2p133 
17 | Rocha CGG, Heidemann ITSB, Souza JB, Durand MK, Maciel KS, Backes MTS

24. Belém JM, Pereira EV, Rebouças VCF, Borges JWP, Pinheiro AKB, Quirino GS. Aspectos teóricos, metodológicos e analíticos de pesquisas etnográficas em enfermagem obstétrica: revisão integrativa. Rev Esc Enferm USP. 2020;54:e03547. doi: 10.1590/s1980-220x2018034203547

25. Carrapato P, Correia P, Garcia B. Determinante da saúde no Brasil: a procura da equidade na saúde. Saúde Soc. 2017;26(3):676-89. doi: 10.1590/S0104-12902017170304

Editora Científica Chefe: Cristiane Cardoso de Paula

Editora associada: Graciela Dutra Sehnem

Fomento: Conselho Nacional de Pesquisa e Desenvolvimento - CNPq

\section{Autor correspondente}

Ivonete Teresinha Schülter Buss Heidemann

E-mail: ivonete.heidemann@ufsc.br

Endereço: Servidão natureza, 160. Bairro Campeche. Florianópolis/ Santa Catarina - Brasil. CEP: 88063 - 545

\section{Contribuições de Autoria}

\section{1 - Carolina Gabriele Gomes da Rocha}

Concepção ou desenho do estudo/pesquisa, análise e/ou interpretação dos dados, revisão final com participação crítica e intelectual no manuscrito.

\section{2 - Ivonete Teresinha Schülter Buss Heidemann}

Concepção ou desenho do estudo/pesquisa, análise e/ou interpretação dos dados, revisão final com participação crítica e intelectual no manuscrito.

\section{3 - Jeane Barros de Souza}

Revisão final com participação crítica e intelectual no manuscrito.

\section{4 - Michelle Kuntz Durand}

Revisão final com participação crítica e intelectual no manuscrito.

\section{5 - Kamila Soares Maciel}

Revisão final com participação crítica e intelectual no manuscrito.

\section{6 - Marli Terezinha Stein Backes}

Revisão final com participação crítica e intelectual no manuscrito.

\section{Como citar este artigo}

Rocha CGG, Heidemann ITSB, Souza JB, Durand MK, Maciel KS, Backes MTS. Social determinants as a way to promote health in prenatal care: perception of primary care professionals. Rev. Enferm. UFSM. 2021 [Accessed in: Year Month Day]; vol.11 e64: 1-17. DOI: https://doi.org/10.5902/2179769264518 